\title{
Comprehensive Care in Pediatric Rheumatic Diseases: A Multifaceted Challenge
}

\section{Kaitlyn L. Gamwell ${ }^{1}$ (D) and Kevin A. Hommel ${ }^{2}$}

Behavior is the most influential predictor for developing a medical condition and subsequently accounts for $40 \%$ of premature deaths ${ }^{1}$. Thus, simplistic approaches to complex health conditions are no longer viable or effective in producing optimal treatment outcomes. Successful psychological interventions have repeatedly demonstrated their profound effect on illness prevention, reduction of disease severity, and improved health-related quality of life $^{2}$. Examples include cognitive behavioral therapy and acceptance and commitment therapy for pain, behavioral interventions to increase healthy lifestyle habits to treat obesity, and self-management support to increase treatment adherence. Despite decades of research, clinical anecdotes, and the World Health Organization's biopsychosocial definition of health as "a state of complete physical, mental and social well-being and not merely the absence of disease or infirmity," ${ }^{3}$ the lack of integrated care models across institutions perpetuates the dearth of behavioral healthcare, contributing to disease morbidity and mortality.

Although society is shifting toward biopsychosocial healthcare approaches, the dualistic mind-body doctrine underlying the biomedical model remains ever-present ${ }^{4}$. The number of epidemics (e.g., obesity, diabetes, addiction) that continue to plague the United States, coupled with the generally lower level of US National Institutes of Health funding appropriated to mental health and pediatric institutes (e.g., National Institute of Mental Health, National Institute of Child Health and Human Development), are testaments to these systemic issues. It is of

This work was supported by a US National Institutes of Health grant (T32 HD068223).

${ }^{1}$ K.L. Gamwell, PhD, Pediatric Psychology Postdoctoral Fellow, Center for Adherence and Self-Management at Cincinnati Children's Hospital Medical Center; ${ }^{2}$ K.A. Hommel, PhD, Professor of Pediatrics, Director, Center for Health Technology Research, Cincinnati Children's Hospital Medical Center, University of Cincinnati College of Medicine, Cincinnati, Ohio, USA.

Address correspondence to K.L. Gamwell, Cincinnati Children's Hospital Medical Center, Division of Behavioral Medicine \& Clinical Psychology, Center for Adherence and Self-Management, 3333 Burnet Ave., MLC 7039, Cincinnati,OH 45229, USA.Email: kaitlyn.gamwell@cchmc.org.
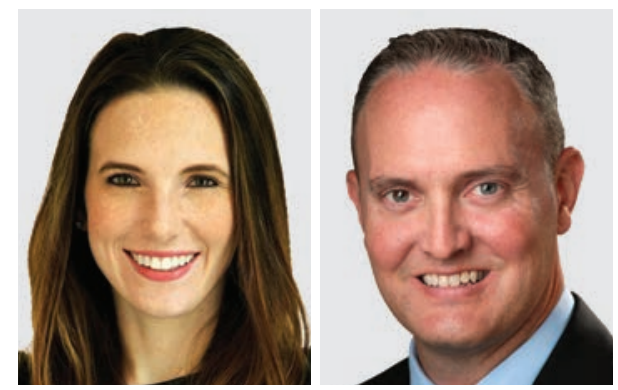

the utmost importance that comprehensive care is provided to promote quality of life for patients and the individuals affected and/or involved in care, particularly in pediatric health conditions $s^{5,6,7}$. However, despite dissemination of research, the translation and application into clinical care often lags behind, leaving many families with unmet needs.

Providing well-rounded treatment for health conditions must be multifaceted ${ }^{8}$, hence the recent trend for multidisciplinary clinics. Yet, what remains less well-integrated is the presence of psychosocial expertise within specialty clinics (e.g., pediatric rheumatology). The factors that contribute to illness prognosis, implementation of and barriers to pediatric self-management, vary across stages of development and involve different people (e.g., parents, teachers, coaches ${ }^{7}$ ). The Pediatric Self-Management Model provides a systematic approach to self-management and the roles of each person involved. This aspirational model further highlights the importance of proactive behavioral healthcare and the efforts needed to increase this initiative in our healthcare system? .

In this issue of The Journal, Rubinstein, et al sought to develop a prioritized research agenda on the mental health needs of youth diagnosed with rheumatic diseases ${ }^{9}$. The authors [members of the Childhood Arthritis and Rheumatology Research Alliance (CARRA) Mental Health Workgroup], used their previous work ${ }^{10,11}$ to highlight comorbid "mental health" areas in the extant pediatric rheumatology literature that warrant further attention and development. The study had several strengths including identification and prioritization of mental health gaps in the literature and an attempt to gain input from patients, parents, and providers on the "importance, feasibility, and actionability" for each mental health domain and domain items. The study also had several limitations, most notably the small sample size $(\mathrm{N}=59)$. Patients and mental health providers were considerably underrepresented $(n=4$ and $\mathrm{n}=3$, respectively). Unfortunately, the inadequate representation of mental health providers is not unique to this study, despite the growing number of pediatric psychologists in the medical field who focus on behavioral health care for chil-

\section{See Mental health research agenda, page 1687}

Personal non-commercial use only. The Journal of Rheumatology Copyright $\subset$ 2020. All rights reserved. 
dren and families affected by chronic conditions and other health-related issues.

The study participants and findings address the disconnect between research promoting the importance of integrated care and the reality of what is being implemented and the resources available ${ }^{12,13}$. For example, the authors call for disease-specific mental health screeners. Yet, there are several validated psychosocial screeners and measures of adjustment for pediatric chronic illness broadly, as well as for rheumatic diseases [e.g., Patient-Reported Outcomes Measurement Information System (PROMIS scales) ${ }^{14}$, Pediatric Quality of Life Inventory (PEDS-QL Rheumatology ${ }^{15}$ )]. Generic behavioral assessments that can be used across chronic conditions are often appropriate, because despite the biological differences in specific illness conditions, the psychosocial experiences and effects are often similar. To the authors' point, indeed, rheumatic disease-specific measures have been beneficial in juvenile idiopathic arthritis and systemic lupus erythematosus, but there are also promising measures for rheumatic diseases collectively and other subgroups that exist ${ }^{16}$. Standardized care is vital to enhance the number of patients receiving quality health services, as is the importance of tailoring care to the needs of the individual. Thus, the concept of illness-specific mental health screeners is valuable, but may not be necessary in all cases, particularly when extant generic assessment tools can be applied appropriately.

Research indicates that youth with chronic health conditions are at risk for poor psychosocial adjustment; in pediatric rheumatic conditions, anxiety and depressive symptoms appear to be the most common ${ }^{17}$. Thus it is not unusual for screeners of depressive and anxiety symptoms and/or disorders to be non-illness-specific because they are assessing symptoms of mental health that do not discriminate by condition. It is more important that information on psychosocial contributors to pediatric rheumatic conditions and use of developmentally appropriate measures be incorporated into rheumatology clinics, than ensuring that they are disease- and subtype-specific. Additional inquiries can be made based on items endorsed as necessary. Further, there are many free self- and proxy-report psychosocial screeners that can be given to caregivers and patients (e.g., Patient Health Questionnaire 9-item scale ${ }^{18}$, Psychosocial Assessment Tool ${ }^{19}$, PEDS-QL generic and Rheumatology ${ }^{15}$, PROMIS; ${ }^{14}$ see Thabrew, et a ${ }^{20}$ for a comprehensive review). Given the downstream effect parental adjustment typically has on child adjustment, it is equally as important to screen caregivers of patients ${ }^{21}$. In sum, there are developmentally appropriate assessment tools readily accessible, a number of which are validated crossculturally and available in multiple languages and conditions.

Understandably, priorities of care remain heavily focused on medical provision, but often neglect behavioral healthcare provision. Hopefully, as we progress so will the incorporation of psychosocial education and screeners into standard care as a requirement for all hospitals and clinics. Unfortunately, the number of medical professionals who are well versed in the psychosocial literature supporting these initiatives is limited. This underscores the importance of multidisciplinary team members when providing care to a patient.

Two related factors relevant to improving behavioral healthcare in subspecialty clinics are board certification and training. The American Board of Pediatrics Specialty Board Examination for Rheumatologists dedicates only $2 \%$ of the examination to competency on psychosocial, developmental, and educational issues ${ }^{22}$. This is reflective of the training focus and indicative of the inherent challenge that exists within pediatric rheumatology (as well as other subspecialty care). Efforts needed to provide better multidisciplinary care include direct behavioral healthcare provision, enhanced training on behavioral healthcare issues so medical providers feel equipped to assess and refer to psychologists or other mental health providers as necessary, and additional institutional resources to allow this shift in care to be made.

Incorporating biopsychosocial models of illness across training and subspecialties would further improve quality of care. For example, well-known models of adjustment including Thompson and Gustafson's Transactional Stress and Coping Model $^{23}$ and the Pediatric Psychosocial Preventative Health Model $^{24}$ provide biopsychosocial conceptualizations of adjustment to chronic conditions from a systems perspective. These models should not only be taught to professionals but also provided to patients to assist in their psychoeducation and empowerment. Thankfully, attempts to address training deficits have begun, most notably in primary care physicians receiving intensive tutelage in integrated health ${ }^{25}$. Additionally, medical schools are beginning to incorporate formal training in these areas, which should have longterm positive effects on patient care. The goal of training is primarily to raise awareness and improve care through recognition of problems. However, integrated care should remain the goal to which all providers aspire because it offers the best and most comprehensive level of care provision.

The specific mental health areas that Rubinstein, et als study highlighted as the most important, actionable, and feasible were (1) determining prevalence and incidence rates of mental health disorders in pediatric rheumatic diseases, (2) determining which are most important to screen for, (3) determining the validity of pediatric rheumatological screeners, and (4) identifying the barriers and facilitators to incorporating mental health screening into pediatric rheumatology settings?. We offer the following additional components as recommended priorities: (1) behavioral health training on the interaction between behavioral and medical health, (2) increasing opportunities for patients and families to receive integrated care, (3) administering psychosocial screeners to caregivers and patients that target internalizing symptoms and disorders using existing validated mental health screeners representing both pediatric rheumatology-specific and generic assessment tools, (4) addressing subclinical psychological symptoms as a means of preventive and proactive care, and (5) a system to facilitate referrals to appropriate behavioral

Personal non-commercial use only. The Journal of Rheumatology Copyright $(\subset) 2020$. All rights reserved. 
health providers if a pediatric psychologist cannot be integrated with the specialty care team.

This editorial is not intended to offer an all-inclusive overview of issues related to providing comprehensive care, but rather to highlight barriers and recent progress, and provide resources to readers invested in improving the quality of life for youth with rheumatic conditions and their families. Rubinstein and colleagues provide valuable insight into much larger systematic issues and initial calls for action'. The authors displayed great initiative by highlighting the importance of multidisciplinary care for pediatric rheumatic diseases, quantifying specific areas that should be prioritized, and providing next steps for researchers and clinicians to address these gaps moving forward. It is our hope that these additional recommendations are also considered.

\section{REFERENCES}

1. McGinnis JM, Williams-Russo P, Knickman JR. The case for more active policy attention to health promotion. Health Aff 2002; 21:78-93.

2. Fisher EB, Fitzgibbon ML, Glasgow RE, Haire-Joshu D, Hayman LL, Kalan RM, et al. Behavior matters. Am J Prev Med 2011;40:e15-e30.

3. World Health Organization. Constitution of the World Health Organization basic document. Geneva (Switzerland): World Health Organization; 1948.

4. Engel GL. The need for a new medical model: a challenge for biomedicine. Science 1977;196:129-36.

5. Chaney JM, Gamwell, KL, Baraldi AN, Ramsey RR, Cushing CC, Mullins AJ, et al. Parent perceptions of illness uncertainty and child depressive symptoms in juvenile rheumatic diseases: examining caregiver demand and parent distress as mediators. J Pediatr Psychol 2016;41:941-51.

6. Drotar D. Promoting comprehensive care for children with chronic health conditions and their families: introduction to the special issue. Childr Serv 2001;4:157-63.

7. Modi AC, Pai AL, Hommel KA, Hood KK, Cortina S, Hilliard ME, et al. Pediatric self-management: a framework for research, practice, and policy. Pediatrics 2012;129:e473-85.

8. Hutchinson TA. Whole person care: transforming healthcare. Cham: Springer; 2017.

9. Rubinstein TB, Ogbu EA, Rodriguez M, Waqar L, Woo JMP, Davis AM, et al. Prioritized agenda for mental health research in pediatric rheumatology from the Childhood Arthritis and Rheumatology Research Alliance Mental Health Workgroup. J Rheumatol 2020;47:1687-95.

10. Davis AM, Rubinstein TB, Rodriguez M, Knight AM. Mental health care for youth with rheumatologic diseases - bridging the gap. Pediatr Rheumatol Online J 2017;15:1-8.

11. Rubinstein T, Davis A, Rodriguez M, Knight A. Addressing mental health in pediatric rheumatology. Curr Treat Options Rheumatol 2018;4:55-72.
12. Wagner EH, Austin BT, Davis C, Hindmarsh M, Schaefer J, Bonomi A. Improving chronic illness care: translating evidence into action. Health Aff 2001;20:64-78.

13. Berry JG, Agrawal RK, Cohen E, Kuo DZ. Special report: the landscape of medical care for children with medical complexity. [Internet. Accessed July 22, 2020.] Available from: www. childrenshospitals.net/cmclitreview

14. Cella D, Yount S, Rothrock N, Gershon R, Cook K, Reeve B, et al. The Patient-Reported Outcomes Measurement Information System (PROMIS): progress of an NIH roadmap cooperative group during its first two years. Med Care 2007;45 Suppl 1:S3-11.

15. Varni JW, Seid M, Smith Knight T, Burwinkle T, Brown J, Szer IS. The Peds $\mathrm{QL}^{\mathrm{m}}$ in pediatric rheumatology: reliability, validity, and responsiveness of the Pediatric Quality of Life Inventory ${ }^{\text {nx }}$ generic core scales and rheumatology module. Arthritis Rheum 2002;46:714-25.

16. Khanna D, Krishnan E, Dewitt EM, Khanna PP, Spiegel B, Hays RD. The future of measuring patient-reported outcomes in rheumatology: Patient-Reported Outcomes Measurement Information System (PROMIS). Arthritis Care Res 2011;63 Suppl 11:S486-90.

17. Williams NA, Burnfield JM. Psychological difficulties and parental well-being in children with musculoskeletal problems in the 2011/2012 National Survey of Children's Health. Rehabil Psychol 2019;64:87-97.

18. Zimmerman M. Using the 9-Item Patient Health Questionnaire to screen for and monitor depression. JAMA 2019;322:2125-6.

19. Kazak AE, Schneider S, Didonato S, Pai AL. Family psychosocial risk screening guided by the pediatric psychosocial preventative health model (PPPHM) using the Psychosocial Assessment Tool (PAT). Acta Oncol 2015;54:574-80.

20. Thabrew H, McDowell H, Given K, Murrell K. Systematic review of screening instruments for psychosocial problems in children and adolescents with long-term physical conditions. Glob Pediatr Heal 2017;4:1-25.

21. Gamwell KL, Mullins AJ, Tackett AP, Suorsa KI, Mullins LL, Chaney JM. Caregiver demand and distress in parents of youth with juvenile rheumatic diseases: examining illness intrusiveness and parenting stress as mediators. J Dev Phys Disabil 2016;28:889-904.

22. Smith RC, Laird-Fick H, D’Mello D, Dwamena FC, Romain A, Olson J, et al. Addressing mental health issues in primary care: an initial curriculum for medical residents. Patient Educ Couns 2014;94:33-42.

23. The American Board of Pediatrics. Pediatric rheumatology content outline: subspecialty in-training, certification, and maintenance of certification (MOC) examinations. [Internet. Accessed June 30, 2020.] Available from: www.abp.org/sites/abp/files/pdf/ pediatric_rheumatology_content_outline.pdf

24. Thompson Jr. RJ, Gustafson KE. Adaptation to chronic childhood illness. Washington, DC: American Psychological Association; 1996.

25. Kazak AE. Pediatric psychosocial preventative health model (PPPHM): research, practice, and collaboration in pediatric family systems medicine. Fam Syst Heal 2006;24:381-95. 